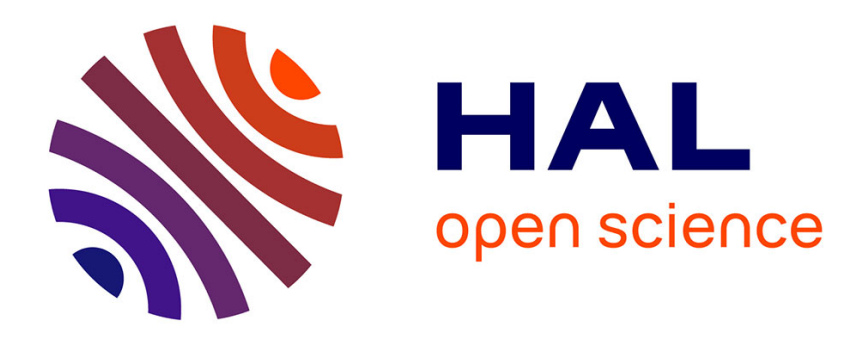

\title{
Le phénomène eurosceptique au Royaume-Uni
}

Agnès Alexandre-Collier

\section{To cite this version:}

Agnès Alexandre-Collier. Le phénomène eurosceptique au Royaume-Uni. Outre-terre. Revue européenne de géopolitique, 2014, 41 (4), pp.100-112. 10.3917/oute1.041.0100 . halshs-01278091

\section{HAL Id: halshs-01278091 https://shs.hal.science/halshs-01278091}

Submitted on 3 Apr 2019

HAL is a multi-disciplinary open access archive for the deposit and dissemination of scientific research documents, whether they are published or not. The documents may come from teaching and research institutions in France or abroad, or from public or private research centers.
L'archive ouverte pluridisciplinaire HAL, est destinée au dépôt et à la diffusion de documents scientifiques de niveau recherche, publiés ou non, émanant des établissements d'enseignement et de recherche français ou étrangers, des laboratoires publics ou privés. 


\section{Le phénomène eurosceptique au Royaume-Uni}

Agnès Alexandre-Collier ${ }^{1}$

Les relations chaotiques entre le Royaume-Uni et l'Union européenne comptent parmi les débats les plus animés de ces quarante dernières années. La vie politique et l'opinion publique, relayées par les médias et la communauté universitaire, offrent le spectacle continu de querelles insurmontables sur les raisons, les enjeux et l'issue possible de cette rivalité. Pour autant, la perspective d'une sortie éventuelle du pays de l'Union européenne, envisagée par le biais d'un référendum d'ici 2017, annoncé par le Premier ministre David Cameron en janvier 2013, ne peut être conçue comme le point final de cette saga dont l'origine est bien antérieure à l'adhésion du pays en 1972. De nombreuses explications sont souvent invoquées mais elles ne permettent pas de comprendre la longévité, voire la radicalisation de ces tensions. Car depuis le milieu des années 2000, le phénomène eurosceptique - expression que nous avons utilisée ailleurs par référence spécifique à la violence des rébellions intrapartisanes engendrée par l'enjeu européen, en particulier au sein du parti conservateur ${ }^{2}$, et qui désigne désormais un véritable mouvement d'opinion - a acquis une nouvelle dimension dont le principal élément déclencheur est la crise que traversent actuellement les pays de la zone euro.

Si l'on se contente de se fier aux résultats des élections européennes de mai 2014 qui ont vu le United Kingdom Independence Party (UKIP), petit parti militant depuis vingt ans pour le retrait du Royaume-Uni de l'Union européenne, recueillir 24 sièges à Strasbourg, la principale manifestation de cette crise, au Royaume-Uni, est une modification profonde du phénomène eurosceptique, néologisme aujourd'hui très euphémisé qui se prête à de multiples définitions. Quand on essaie de structurer ces définitions, on s'aperçoit qu'elles s'articulent autour de deux types de perspectives. $\mathrm{Si}$ on l'appréhende en termes d'intensité, sur un mode plutôt horizontal, l'euroscepticisme en est venu, avec le temps, à désigner non plus une simple réticence, variable et diffuse, au processus politique et monétaire d'intégration européenne mais un vaste mouvement protéiforme d'opposition à l'Union européenne, allant de la mobilisation de certains parlementaires ou individus au sein d'associations et de groupes de pression jusqu'à l'activisme radical de petits partis politiques qui se

\footnotetext{
Professeur de civilisation britannique contemporaine à l'Université de Bourgogne

2 Cf. Agnès Alexandre-Collier, "Le phénomène eurosceptique au sein du parti conservateur britannique ", Politique européenne, $n^{\circ} 6$, hiver 2002, p. 53-73.
} 
sont spécifiquement créés autour de la question de l'adhésion. Sur un axe horizontal, c'est-à-dire en termes d'orientation, permettant d'identifier les différents enjeux qui engendrent l'euroscepticisme, le constat actuel est également celui d'une évolution nette depuis les années 1980-90 quand les eurosceptiques se contentaient de fustiger les politiques communes, comme la Politique Agricole Commune (PAC) et le principe d'une « union sans cesse " plus étroite entre les États-membres, dans des domaines comme la politique monétaire et la création d'une monnaie unique, jusqu'à la période actuelle où l'euroscepticisme désigne désormais un type d'engagement que l'on aurait qualifié il y a vingt ans d' " anti-européen ", c'est-à-dire remettant en cause le principe même de l'appartenance à l'Union européenne.

Ce que révèlent aussi ces définitions, c'est que le phénomène eurosceptique, certes relayé par la presse et un grand nombre d'associations, est avant tout une obsession partisane $^{3}$ : l'enjeu européen divise les grands partis majoritaires en leur sein et est même à l'origine de nouveaux partis comme le UKIP devenu en vingt ans le premier parti britannique au Parlement européen. Cette victoire aux élections européennes de mai 2014 fait suite à des succès croissants aux élections des conseils municipaux et à une série d'élections partielles entre 2011 et 2013. Le UKIP est ainsi arrivé en seconde position dans les circonscriptions de Rotherham, Eastleigh et South Shields, recueillant dans chaque cas plus de $20 \%$ des voix. Alors que les partis majoritaires ne parviennent pas à atténuer leur obsession pour l'Europe, malgré des efforts dans ce sens depuis les élections de mai 2010, la réalité est que l'enjeu européen n'intéresse pas les Britanniques ${ }^{4}$. Mais lorsque ceux-ci sont interrogés plus précisément sur la nature de leur sentiment européen, la réalité est plus complexe. Entre les querelles intrapartisanes dont elle est à l'origine et le souverain mépris dont elle fait l'objet chez les électeurs, l'Europe ne suscite en Grande-Bretagne presque plus que des sentiments négatifs allant de la simple indifférence à la haine la plus farouche. Et l'europhilie est ainsi devenue une maladie honteuse, au point que les partis jadis les plus favorables au projet européen comme les Libéraux-Démocrates ou le Scottish National Party (SNP), plus préoccupé ces derniers temps par le référendum sur l'indépendance de l'Ecosse du 18 septembre 2014, font profil bas.

3 Plus généralement sur les partis politiques britanniques, voir Agnès Alexandre-Collier, Emmanuelle Avril, Les partis politiques en Grande-Bretagne, Paris, Armand Colin, 2013.

4 En moyenne, seuls $4 \%$ des électeurs britanniques considèrent l'Europe comme l'enjeu le plus important pour leur pays, European integration as "the most important issue facing Britain today ", 1997-2011, sondage Ispos-MOR/ consultable en ligne 


\section{LE MAUVAIS ÉLÈVE DE L'EUROPE ?}

Pour expliquer le "malentendu " 5 qui règne entre le Royaume-Uni et l'Europe, un faisceau de causes strictement géographiques (l'insularité), géopolitiques (la relation transatlantique), historiques (la puissance impériale), institutionnelles (la souveraineté du Parlement de Westminster) sont souvent invoqués. Mais ces explications qui associent pêle-mêle des interprétations historiques et constitutionnelles à des stéréotypes culturels ne parviennent pas à rendre compte de la réalité du positionnement britannique dans l'Union européenne et encore moins à expliquer la persistance d'un phénomène, l'euroscepticisme, qui loin de s'essouffler s'est nettement radicalisé.

L'histoire du Royaume-Uni dans l'Union européenne est souvent décrite comme celle d'un partenaire détaché, réticent et peu investi ${ }^{6}$. Cette vision s'explique par une attitude conjuguant l'attentisme caractérisé par une adhésion tardive au Marché commun (1972), principalement motivé par un intérêt économique et commercial, et une évaluation pragmatique des différentes étapes de la construction européenne en termes de coûts et de bénéfices pour le pays. Les refus d'adhérer à l'accord de Schengen comme à l'Union économique et monétaire dont le Royaume-Uni obtient une dérogation au moment des négociations du traité de Maastricht en 1992 figurent souvent parmi les exemples les plus significatifs. S’ajoute à ces événements une volonté continue de négocier sa contribution au budget européen, illustrée à l'origine par l'attitude intransigeante de Margaret Thatcher au sommet européen de Fontainebleau en $1984^{7}$, et le désir d'infléchir l'évolution de l'Union européenne autour d'un modèle européen plus intergouvernemental, libéral et atlantiste, conforme aux intérêts britanniques. À la source de ce modèle il y a la vision churchillienne des trois cercles représentés par le Commonwealth, l'Europe et les États-Unis, au centre desquels la Grande-Bretagne devait garder une position stratégique ${ }^{8}$.

Pour autant, cette histoire ne traduit pas fidèlement la réalité de l'investissement du Royaume-Uni dans la construction européenne. Margaret Thatcher, souvent dépeinte comme la mère spirituelle de l'euroscepticisme britannique, a pourtant engagé son pays dans le marché intérieur en signant l'Acte unique européen en 1986, dont le rédacteur était lui-même un Britannique, le commissaire européen Lord Cockfield. Comme l'explique par ailleurs Pauline Schnapper, l'européanisation a progressivement gagné tous les rouages institutionnels du système administratif, par-

5 Expression empruntée au titre de l'ouvrage de Pauline Schnapper, La Grande-Bretagne et l'Europe : le grand malentendu, Paris, Presses de Sciences Po, 2000.

6 Comme l'indiquent le titre de l'ouvrage de Stephen George, An Awkward Partner: Britain in the European Community, Oxford, Oxford University Press, 1998, $3^{e}$ éd., ou celui de David Gowland, Arthur Turner, Reluctant Europeans. Britain and European Integration (1945-1998), Harlow, Pearson Education, Longman, 2000. C'est nous qui soulignons.

7 Position résumée par la presse sous l'expression légendaire : "I want my money back ».

8 Discours prononcé à Llandudno, 1948. 
lementaire et judiciaire, comme en témoignent le rôle charnière joué par la représentation permanente du Royaume-Uni dans les instances européennes (UK Permanent representation to the EU ou UKREP) ou la création de commissions parlementaires spécifiques, ainsi qu'une application exemplaire des règles européennes ${ }^{9}$, si bien que le pays est parvenu à exercer une influence profonde sur la philosophie générale de la construction européenne autour d'une défense du libre-échange, de la démocratisation des institutions européennes, de l'élargissement à de nouveaux États-membres et d'une défense européenne pro-atlantiste ${ }^{10}$.

Toutefois, depuis la crise financière de 2008 et sous l'influence de la coalition gouvernementale des Conservateurs de David Cameron et des Libéraux-Démocrates de Nick Clegg, ce dernier parti pourtant historiquement très favorable à l'intégration européenne, la trajectoire du Royaume-Uni dans l'Union s'est modifiée. L'objet européen lui-même, cible mouvante dont l'identité ne cesse de diviser les politiques ${ }^{11}$, a profondément changé pour entrer désormais, à l'échelle de la plupart des États-membres, dans l'ère de la méfiance et du désaveu. Les résultats des élections européennes de mai 2014, doublés d'un taux d'abstention record, en sont une triste illustration. Du côté britannique, le veto gouvernemental au pacte de stabilité budgétaire destiné à sauver les pays de la zone euro de la faillite, allié à l'hypothèse d'une sortie de l'Union européenne (British exit ou Brexit) et à la remise en cause du principe même d'appartenance à l'Union, positionnent le pays dans un nouveau schéma où la perception se confond désormais avec la réalité. Le Royaume-Uni est entré dans un nouveau cycle dans lequel le Premier ministre conservateur David Cameron apparaît désormais comme le chantre d'un positionnement plus radical que celui de Margaret Thatcher dont on peut affirmer, rétrospectivement, que son euroscepticisme a plus été une question de style que de substance.

\section{UNE OBSESSION PARTISANE}

L'une des raisons de cette radicalisation réside dans les changements politiques intervenus récemment. Les élections de 2010 ont permis le retour des Conservateurs au pouvoir après treize ans de défaites successives, offrant la possibilité à la nouvelle équipe rassemblée par David Cameron de laisser libre cours à un euroscepticisme stimulé par ces années d'opposition sous les gouvernements New Labour de Tony Blair et Gordon Brown (1997-2010). Car le fait est que l'Europe est devenue une obsession essentiellement partisane, galvanisée par la presse populaire de Rupert Murdoch, propriétaire notamment du quotidien The Sun dont le tirage dépasse les

9 Cf. Pauline Schnapper, Le Royaume-Uni doit-il sortir de I'Union européenne?, Paris, La Documentation française, 2014, p. 90-98.

10 Ibid., p. 99-109.

11 Jacques Delors évoqua l'expression d' "Objet Politique Non Identifié " dans une intervention à Luxembourg du 9 septembre 1985. 
2 millions d'exemplaires, et les multiples organisations qui gravitent autour des partis politiques. Obnubilés par un enjeu qui paradoxalement n'intéresse pas les Britanniques, les partis majoritaires, sans doute influencés par l'ascension du UKIP, affichent une identité idéologique désormais indissociable de la question européenne. Les partis conservateur et travailliste, qui ont toujours été profondément divisés en leur sein, ont subi une évolution remarquable de ce point de vue. Tandis que les Travaillistes avaient, dans les années 1970 et 80, manifesté une hostilité envers ce qu'ils considéraient comme un club capitaliste de riches nations, les Conservateurs apparaissaient à la même époque comme le "parti de l'Europe " ${ }^{12}$. Le référendum du 5 juin 1975 organisé par le gouvernement travailliste de Harold Wilson sur le maintien du pays dans le Marché commun visait surtout à contourner les clivages partisans et à laisser le peuple prendre une décision qui ne faisait pas consensus entre les ministres et députés travaillistes. Du côté conservateur, Edward Heath puis la nouvelle dirigeante du parti élue en février 1975, Margaret Thatcher, firent campagne pour le «oui ». Dans le camp du "non », les opposants conservateurs et travaillistes respectivement sous la houlette d'un Tony Benn à gauche et d'un Enoch Powell à droite se retrouvaient, au-delà des différences partisanes, autour d'une même volonté de défendre la souveraineté nationale incarnée par le Parlement de Westminster. Ces alliances transpartisanes chez les partisans et les opposants au Marché commun révélaient la profondeur des clivages au sein même des partis, alors même que le nouveau parti des Libéraux-Démocrates officiellement créé en 1988 apparaissait déjà comme le seul parti unanimement pro-européen de l'échiquier politique.

Le tournant majeur des années 1990 a été incontestablement la ratification du traité de Maastricht, épisode douloureux qui dura dix-huit mois et se solda par une implosion du parti conservateur, miné de surcroît par des scandales financiers et par une crise du leadership dont John Major, alors Premier ministre, fit les frais. L'échec électoral des Conservateurs en mai 1997, après dix-huit années d'hégémonie, permit la victoire sans précédent du parti travailliste rénové par Tony Blair. Parallèlement, les tensions autour de Maastricht aboutirent à une véritable recomposition du paysage politique dans lequel les partis majoritaires finirent par s'aligner autour de l'enjeu européen : l'euroscepticisme gagna du terrain chez les Conservateurs tandis que les Travaillistes, sans doute par réaction, apparurent comme un parti essentiellement pro-européen ${ }^{13}$. Le style du nouveau Premier ministre, Tony Blair, projetait également l'image d'un pays moins récalcitrant décidé à jouer un rôle au "cœur de l'Europe » sans pour autant nuire à la fameuse " relation spéciale » entretenue avec les États-Unis.

12 Cf. Agnès Alexandre-Collier, La Grande-Bretagne eurosceptique ? L'Europe dans le débat politique britannique, Nantes, Éditions du Temps, 2002.

13 En réalité le parti travailliste était resté également divisé mais la popularité de Tony Blair et le renouvellement de la base parlementaire en 1997, majoritairement acquise aux thèses du New Labour, avait permis au nouveau Premier ministre d'occulter efficacement les divergences. 
C'est également dans le sillage de Maastricht que se sont formés de nombreux groupes de pression et organisations ${ }^{14}$ destinés à poursuivre le combat contre le traité, malgré la ratification parlementaire, et à empêcher toute progression vers une Europe politique et monétaire, dénoncée comme " fédérale ". Le fédéralisme, assimilé par les eurosceptiques britanniques à un super-État centralisateur visant à absorber, voire dissoudre les identités des États constitutifs de cet ensemble, est alors devenu un tabou dans le discours politique, au point que ce qu'ils appelaient le «f-word " était à leurs yeux aussi irrespectueux qu'un mot de cinq lettres. Parmi cette multitude de groupes, la Ligue anti-fédéraliste créée en 1991 par un universitaire de la London School of Economics, Alan Sked, ne tarda pas à changer son nom. Le UKIP naquit ainsi en 1993, évoluant en l'espace de vingt ans d'un simple groupe de pression à un véritable parti politique, doté d'un leader charismatique ${ }^{15}$, Nigel Farage, et d'une machine partisane désormais efficace. Depuis que le UKIP a pour la première fois présenté des candidats aux élections européennes de 1994, il a recueilli 3 sièges en 1999, 13 en 2009 et 24 en 2014. Cette progression fulgurante au Parlement de Strasbourg s'est accompagnée d'une professionnalisation croissante, si bien que le parti d'amateurs composé "de loufoques, de cinglés et de racistes inavoués ", selon les propos de David Cameron, dispose désormais de structures locales bien implantées dans certaines circonscriptions, bénéficie de dons provenant de riches mécènes et de l'aide d'un réseau d'experts en communication politique et de consultants ${ }^{16}$.

Alors que le parti travailliste d'Ed Miliband apparaît aujourd'hui comme un parti critique mais plus ouvert à l'Union européenne que son principal adversaire, martelant des arguments économiques en faveur du maintien dans l'Union, le parti conservateur s'affiche comme le temple d'un euroscepticisme désormais unanime ${ }^{17}$. Le parti reste toutefois divisé mais les divisions ne sont plus les mêmes. Elles révèlent désormais des nuances de degrés entre les partisans d'un euroscepticisme modéré (soft Euroscepticism) favorable au maintien du pays dans l'Union européenne mais hostile à toute intensification du processus d'intégration européenne, et les eurosceptiques radicaux (hard Euroscepticism) qui militent pour le retrait pur et simple du pays ${ }^{18}$. L'élection de David Cameron à la tête du parti le 6 décembre 2005 avait toutefois permis de nourrir l'espoir d'une pacification entre les différentes factions, notamment entre les pro-européens et les eurosceptiques. Le jeune candidat s'était

\footnotetext{
14 Agnès Alexandre-Collier, La Grande-Bretagne eurosceptique?, op. cit., p. 96-100.

15 Le terme est justifié par l'ascension spectaculaire de Nigel Farage qui se targue d'être l'homme politique le plus visionné d'Europe sur YouTube.

16 Cf. Robert Ford, Matthew Goodwin, Revolt on the Right. Explaining Support for the Radical Right in Britain, Abingdon, Routlege, 2014.

17 Même des pro-européens comme Kenneth Clarke ou Stephen Dorrell font désormais profil bas au sein du parti.

18 Cf. Aleks Szczerbiak, Paul Taggart, "Researching Euroscepticism in European Party Systems : A Comparative and Theoretical Research Agenda ", Opposing Europe? The comparative party politics of Euroscepticism, vol. 2, Oxford University Press, 2008, p. 2
} 
officieusement engagé, en échange du soutien des eurosceptiques, à prendre des mesures radicales telles que le retrait des députés conservateurs du groupe du Parti Populaire Européen, décision finalement mise en œuvre en juin $2009^{19}$. De surcroît, les trois quarts des députés conservateurs élus en mai 2010 revendiquaient désormais un euroscepticisme décomplexée ${ }^{20}$. Malgré cette évolution, le curseur de l'euroscepticisme s'est déplacé chez les premiers avec la coalition entre Conservateurs et Libéraux-Démocrates vers une attitude d'hostilité plus nette envers l'Union européenne, ce qui a donné naissance à un nouveau clivage entre modérés et radicaux, axé sur le principe même de l'appartenance à l'Union. Ainsi, David Cameron lui-même a-t-il radicalisé sa position sous la pression conjointe du UKIP et des eurosceptiques radicaux du parti, malgré la présence plus que discrète des Libéraux-Démocrates pro-européens. En octobre 2011, après avoir interdit ${ }^{21}$ à son parti de soutenir une proposition de référendum sur la sortie du Royaume-Uni, il s'est heurté à une rébellion sans précédent de 81 députés conservateurs soit le quart du groupe parlementaire, emmenée par David Nuttall élu en 2010, et soutenue par des pétitions en ligne rassemblant environ 100000 signatures. Dans son discours de Bloomberg du 23 janvier 2013, le Premier ministre, plaidant pour une Europe flexible dans laquelle le pays pourrait bénéficier d'exemptions et renégocier son statut, a finalement annoncé qu’un référendum serait organisé si les Conservateurs étaient réélus en mai 2015. Le rapatriement de compétences nationales qui ont été transférées vers les institutions européennes par le jeu des traités successifs figure parmi les principaux enjeux de cette renégociation que David Cameron envisage avec ses partenaires européens, misant sur son succès pour appeler à voter « oui ». Cette radicalisation eurosceptique est bien le signe d'un changement de perspective qui accorde la priorité aux intérêts partisans. Le Premier ministre est même disposé à passer outre ses soutiens provenant des milieux financiers et du patronat ${ }^{22}$ pour satisfaire ses députés, ses militants et des électeurs séduits par le positionnement plus ferme du UKIP ${ }^{23}$. L'externalisation des enjeux clivants, comme en témoigne également la décision d'organiser un débat sur l'indépendance de l'Écosse, est devenue une stratégie récurrente de David Cameron. Certes courageuse, elle n'en est pas moins dangereuse et fragilise encore

19 Cf. Agnès Alexandre-Collier, Les Habits neufs de David Cameron. Les conservateurs britanniques (1990-2010), Paris, Presses de Sciences Po, 2010.

20 Une étude réalisée par Tim Heppell indique que 76,8 \% des députés conservateurs se déclarent eurosceptiques (50,3\% modérés et 26,5 \% radicaux), cf. Tim Heppell, "Cameron and Liberal Conservatism : Attitudes within the Parliamentary Conservative Party and Conservative Ministers ", The British Journal of Politics and International Relations, vol. 15, 2013, p. 340-361.

21 En imposant un " three-line whip " ou convocation impérative, procédé institutionnel obligeant les députés à suivre les consignes de vote sous peine de sanction, voire d'exclusion temporaire.

22 D'après une enquête réalisée par le patronat (Confederation of British Industry), 8 entreprises sur 10 sont favorables au maintien du pays dans I'Union européenne. Seuls $10 \%$ des hommes d'affaires interrogés souhaitent quitter I'Union. $71 \%$ estiment que l'appartenance à I'Union leur a été économiquement bénéfique, " CBI Survey Shows Firms Want UK to Stay ", Sky News, 12 septembre 2013, <news.sky.com/story/1140581/cbi-survey-shows-firms-want-uk-to-stay-in-eu> [30 juin 2014].

23 Cf. Philip Lynch, Richard Whitaker, "Rivalry on the Right: The Conservatives, the UK Independence Party (UKIP) and the EU issue ", British Politics, vol. 8, n 3, 2013, p. 282-312. 
davantage le Premier ministre déjà éclipsé dans les sondages par le maire de Londres, Boris Johnson, beaucoup plus eurosceptique ${ }^{24}$ et actuel favori des électeurs et sympathisants conservateurs ${ }^{25}$.

\section{LE SYMPTÔME D'UN MALAISE POLITIQUE}

La percée du UKIP dans le paysage politique britannique a révélé un autre phénomène : la montée d'un populisme relativement inédit au Royaume-Uni, arc-bouté sur le rejet de l'Union européenne mais également alimenté par les transformations institutionnelles récemment éprouvées par le premier. Plus largement, on peut même dire comme Chris Gifford que ce populisme est également un symptôme du déclin national et de la crise "post-impériale " que traverse le pays depuis la Seconde Guerre mondiale : "l'opposition à l'intégration européenne ne peut plus être perçue comme un phénomène temporaire et limité de la politique britannique mais doit être considérée comme systémique et essentielle à ce que la Grande-Bretagne est devenue $~_{26}$. Si l'intégration européenne et la mondialisation ont facilité la libre-circulation des personnes et nourri les controverses autour de l'immigration, la dévolution des pouvoirs à l'Écosse et au pays de Galles ${ }^{27}$ introduite en 1997 et la question de l'indépendance de l'Écosse soumise au référendum du 18 septembre 2014 ont engendré un débat sur l'identité du Royaume-Uni dont la souveraineté et l'indépendance subissent désormais des pressions à la fois externes et internes. C'est donc toute la question de la configuration institutionnelle et géopolitique du pays qui se pose, dans le cadre d'un débat plus large sur l'effritement des États-nations européens, consécutif aux processus conjoints d'intégration européenne et de dévolution/décentralisation. L'Europe, bouc émissaire de la crise économique, est aussi la face visible et facilement identifiable d'un malaise plus profond.

En élargissant son programme à la lutte contre l'immigration, et plus spécifiquement à la méfiance envers l'afflux d'immigrés en provenance des pays d'Europe centrale et orientale, le UKIP se présente désormais comme le chantre d'un nationalisme britannique chez qui l'opposition à l'Union européenne n'est plus qu'un élément parmi d'autres. Il n'est pourtant pas le seul à chanter les louanges de la nation britannique. Le British National Party, parti d'extrême droite qui milite plus activement et aussi plus violemment contre l'immigration et l'islam surfe sur la même

24 Cf. Rowena Mason, "Boris Johnson : Britain could have a glorious future outside EU ", The Guardian, 5 août 2014 , $<$ www.theguardian.com/politics/2014/aug/05/boris-johnson-britain-glorious-future-outside-eu> [23 septembre 2014].

25 Cf. Rajeev Syal, "Boris Johnson is public favorite for Conservative leadership - poll ", <www.theguardian.com/ politics/2014/aug/10/boris-johnson-conservative-party-leadership-david-cameron> [23 septembre 2014].

26 Cf. Chris Gifford, The Making of Eurosceptic Britain, Londres, Ashgate, 2014 (à paraître).

27 Transfert de pouvoirs accordés par le Parlement de Westminster au Parlement écossais et à l'Assemblée galloise, nouvellement créés (ou recréés dans le cas écossais). 
vague, si bien que le UKIP est souvent présenté dans les médias comme le pendant « respectable » du BNP. En réalité, le UKIP refuse toute association à l'extrême droite, qui, dans sa version britannique, ne craint pas de véhiculer un discours antisémite et xénophobe. Le rejet d'une alliance avec le Front national de Marine Le Pen, le Parti pour la liberté (PVV) de Geert Wilders, le Parti autrichien de la liberté (FPÖ), le Vlaams Belang belge et la Ligue du Nord italienne en est l'illustration à l'échelle du Parlement européen. Mais son association avec une dissidente du FN, Joëlle Bergeron, souligne dans le même temps l'ambivalence du UKIP vis-à-vis de cette idéologie. Ce dernier cherche également à se démarquer de deux partis, l'English Defence League (EDL) et les English Democrats ${ }^{28}$, qui militent pour la création d'un parlement anglais et sont actuellement l'expression d'un nationalisme proprement anglais. Le mouvement est né d'une réaction à l'encontre du processus de dévolution jugé asymétrique puisque le pays de Galles et l'Écosse disposent actuellement de leurs propres institutions parlementaires, ce qui n'est pas le cas de l'Angleterre qui est pourtant la nation ${ }^{29}$ la plus importante du Royaume-Uni en termes économiques et démographiques. Malgré l'extrémisme de l'EDL associé à des manifestations violentes contre l'intégrisme musulman, une alliance temporaire avec un petit parti, le British Freedom Party, dirigé par un ancien membre du UKIP montre que la stratégie de différenciation avec un nationalisme radical est loin d'être simple. De surcroît, sa popularité aux élections des conseils municipaux du Sud-Est de l'Angleterre tend à assimiler le nationalisme revendiqué par le UKIP à un repli frileux sur cette nation, à une vision étriquée de "l'Angleterre profonde " (Little England) dont il se défend pourtant.

De fait, le succès du UKIP doit s'interpréter dans une perspective bien plus large, comme le symptôme d'un malaise qui affecte bon nombre de pays européens. Cette crise du politique ressort avec encore plus de virulence dans un pays présenté comme le berceau de la démocratie parlementaire et qui s'affiche encore comme un modèle de stabilité et de modération. Le contraste avec la désaffection des Britanniques qui, encore fiers de leur propre système, en viennent à déplacer leur malaise vers les institutions européennes n'en étant que plus aigu. Stigmatisées pour leur bureaucratie abusive, leur déficit démocratique et l'excès de centralisation et de réglementation, les institutions européennes, cibles des critiques, sont perçues comme l'exact contraire d'un système qui jusqu'à présent fonctionnait comme un modèle intouchable. Or, ce que révèle la percée du UKIP ce sont aussi les premières fissures de ce système britannique en apparence infaillible. Si le fonctionnement même des institutions

28 Cf. Agnès Alexandre-Collier, Emmanuelle Avril, Les partis politiques en Grande-Bretagne, op. cit., p. 197-198.

29 Le terme de nation, et non de région, est presque systématiquement utilisé pour qualifier l'Angleterre et surtout l'Écosse, le pays de Galles et I'Irlande du Nord, dotés d'identités distinctes de celle de l'Angleterre et d'une histoire spécifique, le Royaume-Uni étant l'union de nations historiquement indépendantes unies à l'Angleterre à l'issue de traités successifs appelés " actes d'union". 
politiques britanniques n'est pas remis en cause ${ }^{30}$, les acteurs de ces institutions font l'objet d'une méfiance nouvelle, notamment depuis le scandale des notes de frais de mai 2009. Celui-ci a mis en évidence une corruption dont la classe politique n'était pas exempte, malgré une tendance persistante chez les parlementaires britanniques à associer ce phénomène aux pays du continent européen. Une partie du programme du UKIP s'articule désormais autour d'un nouveau populisme ${ }^{31}$ qui incarne le divorce entre les Britanniques et la classe politique traditionnelle et plaide pour un renouvellement des acteurs politiques en faveur d'individus qui ne se targueraient plus d'être des professionnels de la politique mais des citoyens ordinaires. Le succès de Nigel Farage est aussi celui de l'homme de la rue qui méprise l'omniprésence du politiquement correct, d'un excentrique connu pour son goût de la controverse et ses boutades ${ }^{32}$, qui milite pour le retour du tabac dans les pubs, la dépénalisation des drogues douces et contre tout ce qu'il considère comme une entrave aux libertés individuelles. Poussé à l'extrême, le UKIP cultive un goût prononcé pour un libéralisme économique et culturel qui s'illustre dans une méfiance profonde envers l'État, une vision libertarienne s'apparentant en ce sens au thatchérisme le plus radical. L'extension de la pratique référendaire à grande échelle, souhaitée par le parti, est le signe d'une volonté de mettre en avant une nouvelle pratique politique qui privilégierait une proximité directe avec le peuple aux dépens du modèle de démocratie parlementaire encensé par ses représentants à la Chambre des Communes. Le succès de Nigel Farage s'explique aussi par l'attrait qu'il exerce auprès des ouvriers ${ }^{33} \mathrm{car}^{\prime} \mathrm{s}^{\prime} \mathrm{l}$ est fréquent d'évoquer la concurrence entre les Conservateurs et le UKIP, la réalité est aussi celle d'une popularité croissante du UKIP dans les bastions travaillistes, qui en fait un rival de plus en plus dangereux, même si pour le moment le mode de scrutin majoritaire uninominal à un tour aux élections législatives lui interdit l'accès au Parlement de Westminster. Le 28 août 2014, la défection du député conservateur Douglas Carswell, figure du proue de la fronde eurosceptique, qui a rejoint le UKIP et devrait être réélu dans sa circonscription sous cette nouvelle étiquette, représente un espoir inédit pour ce petit parti de parvenir d'autres députés du parti de David Cameron et de faire ainsi son entrée au Parlement britannique aux élections de mai 2015.

30 Le 5 mai 2011, les Britanniques consultés par référendum ont rejeté à 67,9\% l'idée d'un changement du mode de scrutin (majoritaire uninominal à un tour).

31 Cf. Agnès Alexandre-Collier, "Populismes ordinaires au Royaume-Uni ", in Marie-Claude Esposito, Alain Laquièze, Christine Manigand (éd.), Populismes. L'envers de la démocratie, Paris, Vendémiaire, 2012, p. 133-141.

32 Attribuant par exemple à Herman Van Rompuy " le charisme d'une serpillière humide et l'aspect d'un petit guichetier de banque ".

33 Cf. Robert Ford, Matthew Goodwin, Revolt on the Right, op. cit. 


\section{CONCLUSION}

Le Royaume-Uni va-t-il sortir de l'Union européenne ? L'euroscepticisme aurat-il gagné ? Cette question est aujourd'hui sur toutes les lèvres mais il est impossible d'y répondre, tant elle dépend entièrement du contexte politique et économique à la fois britannique et européen qui entourera le référendum, si celui-ci a bien lieu, de la formulation de la question posée et de l'organisation de la campagne qui sera menée dans les deux camps. L'euroscepticisme ne cesse toutefois de progresser, offrant l'image d'un mouvement de plus en plus puissant mais encore peu structuré en tant qu'ensemble. Le camp du "non " rassemble déjà des groupes à la fois anciens, comme le groupe de Bruges, et plus récents comme Better Off Out, Open Europe, the Fresh Start Project, auxquels s'ajoutent depuis l'annonce du référendum I Want a Referendum, Vote UK Out of Europe ou encore The People's Pledge. Mais tous les facteurs préalablement énoncés constituent un faisceau d'incertitudes qui ouvrent la voie à des hypothèses fantaisistes sur l'avenir. Peut-on véritablement, à trois ans de l'éventuel scrutin, se fier aux sondages actuels qui tendent à démontrer la probabilité d'un Brexit? Un sondage récent de juin 2014 indique en effet que $48 \%$ des Britanniques interrogés souhaitent quitter l'Union européenne contre $37 \%$ et $15 \%$ qui ne se prononcent $\mathrm{pas}^{34}$. En réalité, lorsque le sondage est plus précis et porte sur l'hypothèse d'une sortie une fois que le gouvernement sera parvenu à renégocier avec ses partenaires européens les conditions de son appartenance, les chiffres sont très différents : $57 \%$ des Britanniques interrogés déclarent alors vouloir rester dans l'Union contre $22 \%$ et $16 \%$ d'indécis ${ }^{35}$.

Les scénarios ne manquent pas pour parer à toutes les éventualités ${ }^{36}$. Les partisans d'un Brexit soutiennent que la sortie de l'Union européenne permettrait l'accès à d'autres marchés pour le Royaume-Uni qui continuerait toutefois à bénéficier d'un système de libre-échange avec l'Union. Le Royaume-Uni pourrait ainsi renforcer les liens avec ses anciennes colonies, aujourd'hui membres du Commonwealth, comme l'Inde. Le pays serait libéré des contraintes juridiques et administratives imposées par les traités et la réglementation européenne. Surtout, les économies que représenterait la fin de la contribution britannique au budget européen ou des frais liés à la transposition des directives européennes constitueraient un gain considérable. Les opposants à la sortie de l'Union soulignent quant à eux la perte de l'immense marché que représente l'Union européenne qui constitue aujourd'hui $46 \%$ des exportations et $51 \%$ des importations britanniques. Ils insistent également sur la dégradation des relations avec les États-Unis qui eux soutiennent fermement la présence britan-

34 Sondage Opinium pour The Observer, 19-20 juin 2014.

35 Sondage YouGov pour The Sun, 15-16 juin 2014.

36 Les quelques exemples développés ci-dessous renvoient à l'ouvrage de Pauline Schnapper, Le Royaume-Uni doit-il sortir de l'Union européenne?, op. cit. Pour plus de détails se référer aux pages 142-145. 
nique dans l'Union et redoutent l'affaiblissement diplomatique du Royaume-Uni au sein des structures comme l'Otan. Car comme le souligne Pauline Schnapper, "l'influence que peut exercer le Royaume-Uni au sein des organisations internationales, y compris l'Otan, aux États-Unis, en Chine, en Inde et ailleurs, est liée au fait qu'il est membre de l'Union européenne ${ }^{37}$. Mais le grand perdant serait, pour eux, la City de Londres, première plateforme financière européenne $e^{38}$, qui pourrait craindre un exode des entreprises financières vers Wall Street notamment. Cette position révèle dans une certaine mesure toute l'ambivalence de la position même du gouvernement, notamment de David Cameron et de son chancelier de l'Échiquier, George Osborne, qui se déclarent par exemple favorables à l'évolution de la zone euro vers une union fiscale, non pas parce qu'ils souhaitent que le pays fasse partie de la zone euro, mais parce qu'ils redoutent les conséquences de sa désintégration pour l'économie et le système bancaire britanniques ${ }^{39}$. Avec un budget de l'UE diminué de $11 \%{ }^{40}$, ils soulignent également les répercussions sur l'Union en tant que telle, économiquement et militairement très affaiblie par ce départ ${ }^{41}$ et certainement encore plus discréditée qu'elle ne l'est déjà dans l'opinion publique, non seulement au Royaume-Uni mais dans de nombreux autres pays européens. En se conjuguant à l'hypothèse d'un vote positif au référendum sur l'indépendance de l'Écosse de septembre 2014 - spectre brandi encore quelques jours avant le scrutin - les pronostics les plus alarmistes décrivaient déjà le scénario inquiétant d'un pays démembré et isolé, réduit à la seule Angleterre, dépossédé de toute influence. Or, malgré la victoire du « oui » au référendum écossais (55,3\% contre 44,7\% avec un taux de participation record de 84,6\%), le scénario d'une future Écosse indépendante et le spectre du démembrement national n'ont pas pour autant disparu.

37 Ibid., p. 145.

38 Les services financiers représentent $10 \%$ du PIB britannique.

39 Cf. Andrew Gamble, "Better Off Out ? Britain and Europe ", The Political Quarterly, vol. 83, n 1, 2012, p. 471.

40 Le Royaume-Uni est en effet le quatrième contributeur net derrière I'Allemagne, la France et I'Italie.

41 Pauline Schnapper, Le Royaume-Uni doit-il sortir de I'Union européenne ?, op. cit., p. 146-147. 
Majorités aux élections européennes de mai 2014

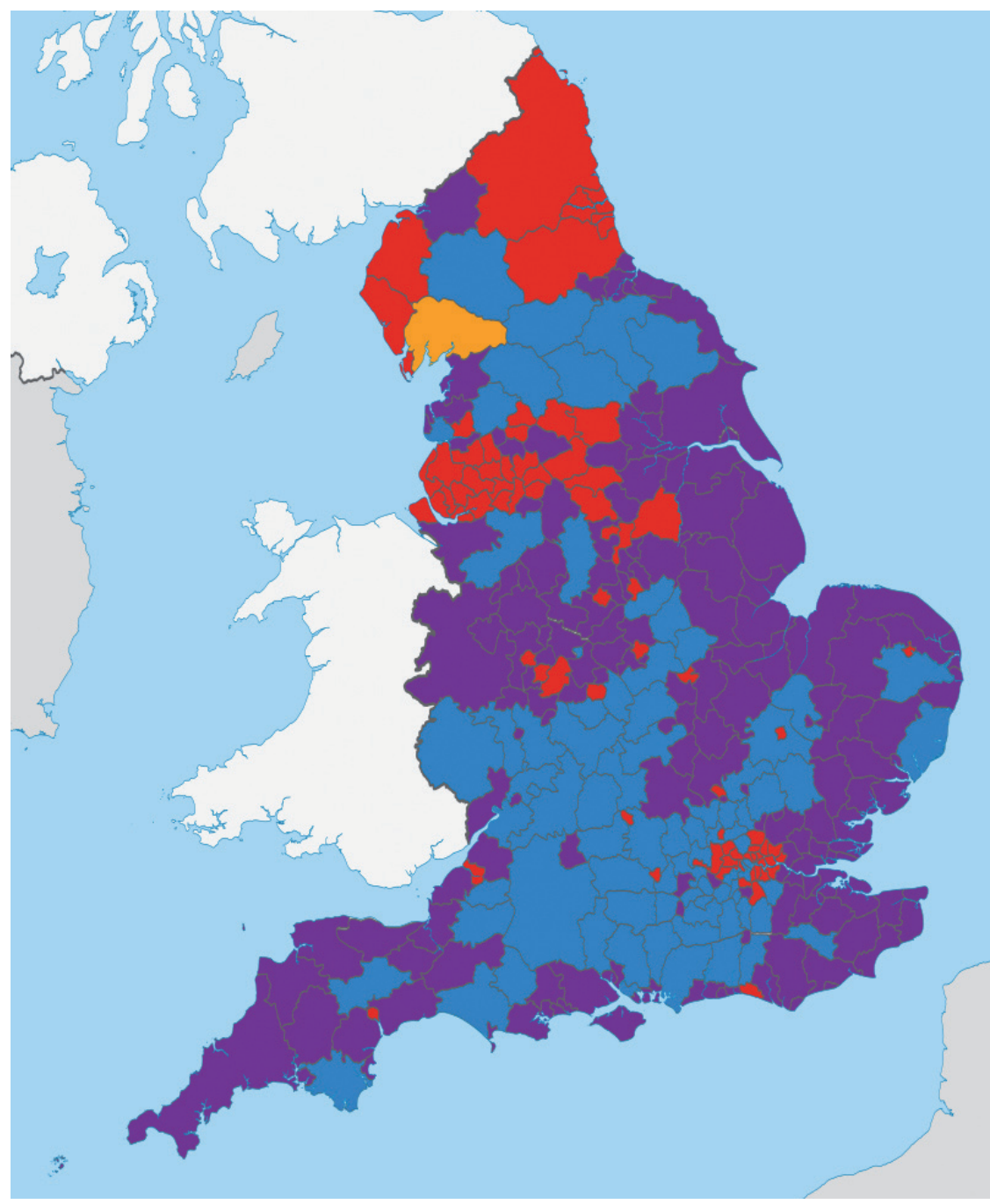

